\title{
Research on High-tech Ammunition Training System Based on Virtual Reality Technology
}

\author{
Zhang Meng, Xu Lutie, Yu Weibo, Liu Hongbo
}

\begin{abstract}
With the development of computer technology, the virtual reality technology has become more and more widely used in military field. The training of high-tech ammunition has the problems of low efficiency, high cost, poor security and inconsistent working mechanism. This paper fully analyzes the construction objectives and main contents of the high-tech ammunition virtual training, and applies virtual reality technology and Vega Prime to achieve the simulation of the process of a rocket weapon system to combat the target. This paper also makes further research on the technology of rocket trajectory calculation, real-time collision detection, etc., and achieves the whole process of combat simulation of the weapon system. The establishment of the system opened a new dimension of the military ammunition combat training .
\end{abstract}

\section{Introduction}

Currently, there are several methods of the training of large complex ammunition equipment system as follows: Firstly, learning the relevant theories through pictures, videos, animation or multimedia resources,etc.; Secondly, strengthening the theoretical understanding and securitying skills via internship and practical training such as manipulating ammunition models and their main components; Thirdly, understanding the combat applications and combat security of ammunition by observing in troops.Due to the limitations of equipment, cost, safety risk and other teaching conditions, there are only scant chances for students to carry on live warfare program training ${ }^{[1]}$. Although certain training effect can be obtained from this kind of training mode, its defects in many aspects, such as reproducing the training process, simulating small probability events, showing the actual scene and self-training still exist. Students can not correct cognitive errors on their own initiative, besides, their abilities to deal with emergencies and psychological endurance for harsh battlefield environment can' $t$ get equipped or improved ${ }^{[2]}$. Solving the various problems and contradictions mentioned above has become problems that need to be solved urgently for ammunition combat operations and combat security research institutions.

The application of virtual reality technology in ammunition training is a new way to solve the contradictions above. Since the technology possesses abilities to simulate ammunition combat environment and smoke light effects and sense the relative position and attitude of the operator/ammunition in the characteristic environment, it is very suitable for the combat operation and security training of ammunition equipment. This technique not only allows the trainees to master the equipment theory and combat skills before training for confrontation ${ }^{[3-5]}$, but also minimizes the actual operation accident caused by personal factors, holding great potential to achieve the ideal training effect and the best military economic benefits.

\section{Function and Composition of Ammun-i tion Virtual Reality System}

\subsection{The goal of the system}

The objective of this system is to complete a integrated dynamic simulation system of the structure, component status, ammunition emission, ammunition movement of a rocket weapon system. Data and status are graphically described entirely, which can be tested, improved, and evaluated for technical and tactical performance ${ }^{[6]}$.

\subsection{Function of the system}

The main function of the system is to simulate the whole process of a rocket weapon system to hit the target with the command car commanding the launch vehicle to aim and launch after locating the target position under the command of the simulation control node. Here are the specific processes:

1) The simulation control node is responsible for controlling the signal generation;

2) It generates a control signal and transmits it to the weapon system;

3) Completing the simulation of the process includes rocket loading, barrel lift, gunpowder burning and launch, etc. based on the control command and physical model.

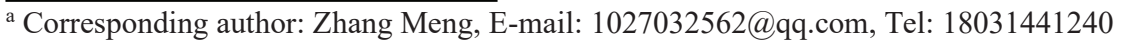


4) Completing the rocket shooting under the comman-d of control signal;

5) Completing the simulated scenes and effects display of all the process;

6) Carrying out the dynamic observation process of profile;

7) It possesses multi-window display function.

The system performer is the user and the simulation control node, which provide the information or issue instructions to the system respectively, and the system provides operational results based on them ${ }^{[7-9]}$.

\subsection{The composition of the system}

The logical structure of a rocket weapon system simulation based on Vega Prime is shown in Fig.1, and the diagram includes a simulation control node and a simulation demonstration node, which communicate by UDP protocol communication via the network connection. The simulation demonstration node consists of network management and transmission module, analog signal simulation module, visual simulation module, physical simulation module, database processing module and result visualization module.

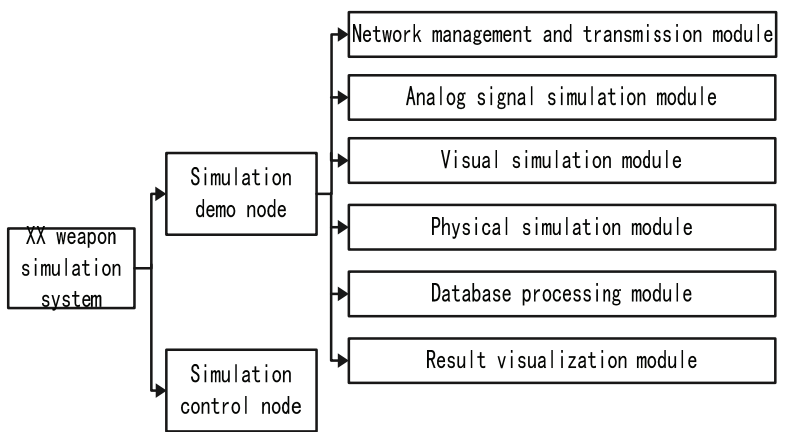

Figure 1. The structure of system

\section{The key technology of the system}

The effect of the simulation system depends mainly on the authenticity of the system motion model. The motion model of the system mainly includes rocket flight and collision detection, etc ${ }^{[10]}$.

\subsection{Trajectory model}

\subsubsection{Coordinate system and conversion relationshi- $p$}

For the convenience of the theoretical research and simulation of the rocket's motion in the inertial space, this paper establishes the emission coordinate system $O x y z$, the missile coordinate system $O x_{1} y_{1} z_{1}$, the quasi-missile coordinate system $O x_{4} y_{4} z_{4}$, the quasi-velocity coordinate system $O x_{v} y_{v} z_{v}$ and the trajectory coordinate system $O x_{2} y_{2} z_{2}$. And the Earth coordinate system $o_{e} x_{e} y_{e} z_{e}$ and 84 coordinate system WGS-84 are also be utilized in order to meet the need of guidance simulation $^{[11]}$.
For the convenience of analysis, coordinates need to be converted, commonly used are:

(1) The conversion matrix from $o_{e} x_{e} y_{e} z_{e}$ to $o x y z$

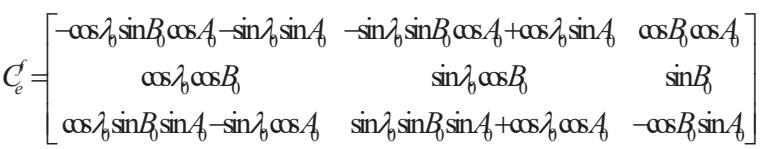

Then conversion relationship of the speed vector from $o_{e} x_{e} y_{e} z_{e}$ to $o x y z$ is:

$$
\left[\begin{array}{c}
V_{x} \\
V_{y} \\
V_{z}
\end{array}\right]=C_{e}^{f}\left[\begin{array}{c}
V_{x e} \\
V_{y e} \\
V_{z e}
\end{array}\right]
$$

The transformation of the position coordinate component from $o_{e} x_{e} y_{e} z_{e}$ to $o x y z$ is:

$$
\left[\begin{array}{l}
x \\
y \\
z
\end{array}\right]=C_{e}^{f}\left[\begin{array}{c}
x_{e}-R_{x e 0} \\
y_{e}-R_{y e 0} \\
z_{e}-R_{z e 0}
\end{array}\right]
$$

Where:

$$
\begin{aligned}
& \left\{\begin{array}{l}
R_{x e 0}=\left(N+h_{0}\right) \cos B_{0} \cos \lambda_{0} \\
R_{y e 0}=\left(N+h_{0}\right) \cos B_{0} \sin \lambda_{0} \\
R_{z e 0}=\left(N\left(1-e^{2}\right)+h_{0}\right) \sin B_{0}
\end{array}\right. \\
& N=a_{e} / \sqrt{1-e^{2} \sin ^{2} B_{0}}
\end{aligned}
$$
geocentric vector at launch point in the earth coordinate system;

$x_{e}, y_{e}, z_{e}-$ The component of geocentric vector at the centroid of the rocket in the earth coordinate system;

$N$ - The radius of prime circle of launch point.

(2) The conversion matrix from $o x_{1} y_{1} z_{1}$ to $o x y z$ is: $C_{1}^{\prime}=\left[\begin{array}{ccc}\cos \phi \cos \psi & -\sin \phi \cos \psi \cos \gamma+\sin \psi \sin \gamma & \sin \phi \cos \psi \sin \gamma+\sin \psi \cos \gamma \\ \sin \phi & \cos \phi \cos \gamma & -\cos \phi \sin \gamma \\ -\cos \phi \sin \psi & \sin \phi \sin \psi \cos \gamma+\cos \psi \sin \gamma & -\sin \phi \sin \psi \sin \gamma+\cos \psi \cos \gamma\end{array} \mid(6)\right.$

(3) The conversion matrix from $o x y z$ to $O x_{4} y_{4} z_{4}$ is:

$$
C_{f}^{4}=\left[\begin{array}{ccc}
\cos \phi \cos \psi & \sin \phi & -\cos \phi \sin \psi \\
-\sin \phi \cos \psi & \cos \phi & \sin \phi \sin \psi \\
\sin \psi & 0 & \cos \psi
\end{array}\right]
$$

(4) The conversion matrix from $o x_{4} y_{4} z_{4}$ to $O x_{1} y_{1} z_{1}$ is:

$$
C_{4}^{1}=\left[\begin{array}{ccc}
1 & 0 & 0 \\
0 & \cos \gamma & \sin \gamma \\
0 & -\sin \gamma & \cos \gamma
\end{array}\right]
$$

\subsubsection{Rocket dynamic equation}

(1) The kinematic equation of centroid motion of the rocket established in the emission coordinate system is:

$$
\left[\begin{array}{l}
\frac{d x}{d t} \\
\frac{d y}{d t} \\
\frac{d z}{d t}
\end{array}\right]=\left[\begin{array}{l}
V_{x} \\
V_{y} \\
V_{z}
\end{array}\right]
$$


(2) The angular velocity equation of the rocket in the quasi-missile coordinate system is:

$$
\left[\begin{array}{c}
\frac{d \gamma}{d t} \\
\frac{d \psi}{d t} \\
\frac{d \varphi}{d t}
\end{array}\right]=\left[\begin{array}{c}
\omega_{x 4}-\omega_{y 4} \operatorname{tg} \varphi \\
\frac{\omega_{y 4}}{\cos \varphi} \\
\omega_{z 4}
\end{array}\right]
$$

(3) The kinetic equation of centroid motion in the coordinate system is:

$$
\left[\begin{array}{l}
\frac{d V_{x}}{d t} \\
\frac{d V_{y}}{d t} \\
\frac{d V_{z}}{d t}
\end{array}\right]=\frac{1}{m}\left[\begin{array}{l}
F_{x}+F_{l k x}+F_{c x}+F_{g x}+R \\
F_{y}+F_{l k y}+F_{c y}+F_{g y}+R_{y} \\
F_{z}+F_{l v z}+F_{c z}+F_{g}+R_{z}
\end{array}\right]+\left[\begin{array}{l}
g_{x} \\
g_{y} \\
g_{z}
\end{array}\right]+\left[\begin{array}{l}
a_{c x} \\
a_{c} \\
a_{c z}
\end{array}\right]+\left[\begin{array}{l}
a_{c x} \\
a_{e y} \\
a_{c z}
\end{array}\right]
$$

(4) The kinetic equation of the centroid rotation established in the quasi-coordinate system is:

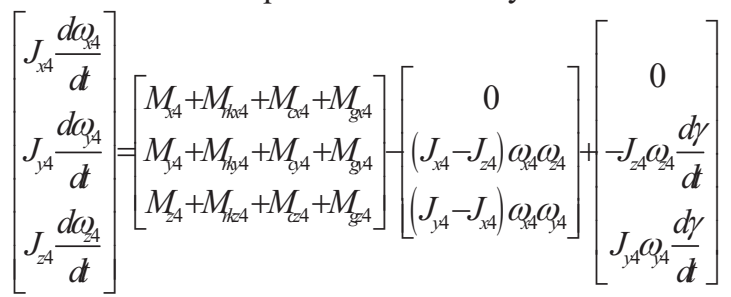

(5) Geometric relationship equation. Assuming the vertical component of wind is 0 , the Mach number equation of the rocket relative to the atmospheric speed and dynamic pressure is:

$$
\begin{gathered}
{\left[\begin{array}{l}
W_{x} \\
W_{y} \\
W_{z}
\end{array}\right]=\left[\begin{array}{c}
-W \cos \left(A_{0}-A_{w}\right) \\
0 \\
W \sin \left(A_{0}-A_{w}\right)
\end{array}\right]} \\
{\left[\begin{array}{l}
V_{x w} \\
V_{y w} \\
V_{z w}
\end{array}\right]=\left[\begin{array}{l}
V_{x} \\
V_{y} \\
V_{z}
\end{array}\right]-\left[\begin{array}{l}
W_{x} \\
W_{y} \\
W_{z}
\end{array}\right]} \\
V_{w}=\sqrt{V_{x w}^{2}+V_{y w}^{2}+V_{z w}^{2}} \\
M_{a w}=\frac{V_{w}}{\sqrt{1.4 \times 287.053 T_{H}}} \\
q=\frac{1}{2} \rho_{H} V_{w}^{2}
\end{gathered}
$$

Convected acceleration and Coriolis acceleration due to the objective existence of the earth rotation when the rocket is launching:

$$
\begin{gathered}
{\left[\begin{array}{l}
a_{e x} \\
a_{e y} \\
a_{e z}
\end{array}\right]=\left[\begin{array}{ccc}
\omega_{e}^{2}-\omega_{e x}^{2} & -\omega_{e x} \omega_{e y} & -\omega_{e x} \omega_{e z} \\
-\omega_{e x} \omega_{e y} & \omega_{e}^{2}-\omega_{e y}^{2} & -\omega_{e y} \omega_{e z} \\
-\omega_{e x} \omega_{e z} & -\omega_{e y} \omega_{e z} & \omega_{e}^{2}-\omega_{e z}^{2}
\end{array}\right]\left[\begin{array}{l}
R_{x d} \\
R_{y d} \\
R_{z d}
\end{array}\right]} \\
{\left[\begin{array}{l}
a_{c x} \\
a_{c y} \\
a_{c z}
\end{array}\right]=\left[\begin{array}{ccc}
0 & 2 \omega_{e z} & 2 \omega_{e y} \\
2 \omega_{e z} & 0 & 2 \omega_{e x} \\
2 \omega_{e y} & 2 \omega_{e x} & 0
\end{array}\right]\left[\begin{array}{l}
V_{x} \\
V_{y} \\
V_{z}
\end{array}\right]}
\end{gathered}
$$

In the formula above: $\mathrm{F}$ is the engine thrust; $\mathrm{Rx} 4$ 、 Ry4、Ry4 is the aerodynamic; gx 、 gy gz is the gravity; Frkx、Frky、Frkz is the Coriolis force of the gas stream; Fcx1、Fcy1、Fcz1 is the pulse control force; Fgx 、 Fgy,$F g z$ is the interference force;Mx4、My4、Mz4 is the aerodynamic moment;Mrkx4、Mrky4、Mrkz4 is the Coriolis moment of gas flow;Mcx1、Mcy1、Mcz1 is the control torque; Mgx4、Mgy4、Mgz4 is the interference torque;

\subsection{Collision detection model}

BUMP is a collision detection method based on the bounding box. In the simulation system, Since Vega Prime is a frame-based operation, the rocket motion trajectory is a motion curve composed of many discrete points. When the flight speed exceeds the threshold value, the target may locate between the two points, and the system can not detect the collision. Therefore, the BUMP method must be improved. First, it is necessary to calculate the shape change of the bounding box, and transform matrix $\mathrm{T}$.

$\mathrm{T}=\mathrm{T}_{\mathrm{rx}} \mathrm{T}_{\mathrm{ry}} \mathrm{T}_{\mathrm{rz}}=\left[\begin{array}{cccc}1 & 0 & 0 & 0 \\ 0 & \cos \theta & \sin \theta & 0 \\ 0 & -\sin \theta & \cos \theta & 0 \\ 0 & 0 & 0 & 1\end{array}\right] \cdot\left[\begin{array}{cccc}\cos \phi & 0 & -\sin \phi & 0 \\ 0 & 1 & 0 & 0 \\ \sin \phi & 0 & \cos \phi & 0 \\ 0 & 0 & 0 & 1\end{array}\right] \cdot\left[\begin{array}{cccc}\cos \rho & \sin \varphi & 0 & 0 \\ -\sin \varphi & \cos \rho & 0 & 0 \\ 0 & 0 & 1 & 0 \\ 0 & 0 & 0 & 1\end{array}\right]$

In order to improve efficiency and accuracy, the method used in this system is: No collision detection is performed before hitting the target. If the distance vector angle between current frame and next frame is greater than right angle, take several points on the track for bounding box collision detection. If there is no collision, target is not hitted. This method needs less amounts of computation and is relatively accurate ${ }^{[12]}$.

$\mathrm{S}_{\mathrm{h}}$ is the centroid of the rocket, $\mathrm{b}\left(\mathrm{S}_{\mathrm{h}}\right)$ is bounding box of object, $\mathrm{A}$ is the vector from the rocket to target, The algorithm is as follows:

1) Calculating the current frame vector $A_{c}$

2) Calculating the next frame vector $A_{n}$ according to the frame interval

3) If $A_{c} \cdot A_{n} \leq 0$, take several points on the track between the two frames and calculate the $b\left(\mathrm{~S}_{\mathrm{h}}\right)$

4) If (exist $b($ Sh)! $=\Phi)$, turn on effects

5) Else do not turn on special effects (Did not hit the target)

6) If $A_{c} \cdot A_{n} \geq 0$, then $A_{c}=A_{n}$

7) Goto 2)

During the flight of the missile, the system compares the methods before and after the improvement. The results show that the possibility of detecting the collision by the former method is inferior in the state of high-speed flight. In 50 experiments, crossing phenomenon was happened. While using the improved methods, crossing phenomenon did not occur in all the 50 experiments, which means the success rate was $100 \%$.

\section{Design and implementation of the system}




\subsection{Realization of rocket flight process}

Rocket flight process can be divided into several stages include uncontrolled section, free flight section, closedloop display guidance, proportional guidance, etc. The simulation of the rocket on the trajectory is shown in Fig.2 and Fig.3.

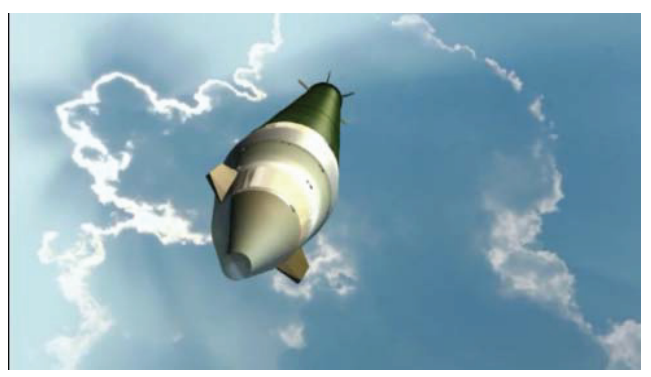

Figure 2. The process of rocket movement

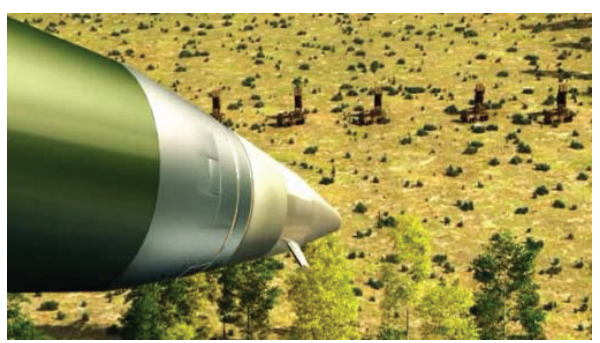

Figure 3. The process of rocket movement

In order to observe the battlefield thoroughly, the simulation system used the form of picture-in-picture to monitor. Users can switch between different windows as needed. Flight attitude and trajectory of rocket can be obtained from rocket tracking window. The target tracking window is mainly responsible for monitoring the trajectory of the target, the collision between the rocket and the target, and the degree of damage after the target is hitted.

\subsection{Realization of hitting target}

Rocket gun simulation is the key part of the weapon system simulation, The initial state, flight trajectory, placement of every rocket projectile should be calculated separately. In order to standardize the movement of rockets, the system defines a structure that contains the spatial motion attributes and control parameters of the bomb.

typedef struct gun_shot I

double timeout;

//rocket life

double flight_time;

//flight duration

float $\mathrm{x} 0, \mathrm{y} 0, \mathrm{z} 0, \mathrm{~h} 0, \mathrm{p} 0, \mathrm{r} 0 ; / /$ position

float $\mathrm{Vx}, \mathrm{Vy}, \mathrm{Vz} ; \quad / /$ the speed of the rocket

float az;

//rocket Acceleration

float starttime:

int state;

//starting time

//status

vpIsector * los;

//impact checking

vpCommon*handle; //rocket handle

vpPosition* pos;

//Position parameter
As the rockets hit different targets with different exploding effects and destructive consequences, the system sets different eigenvalues to islands and trees, etc. In the rocket collision detection, the system will determine the special effects and damage results caused by eigenvaluse based on the results.

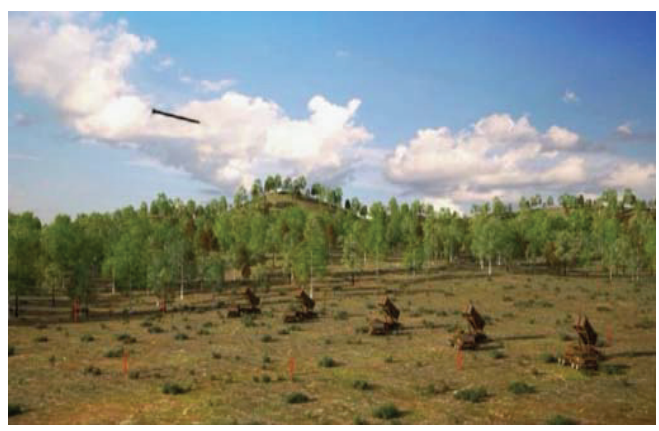

Figure 4. The simulation of rocket trajectory

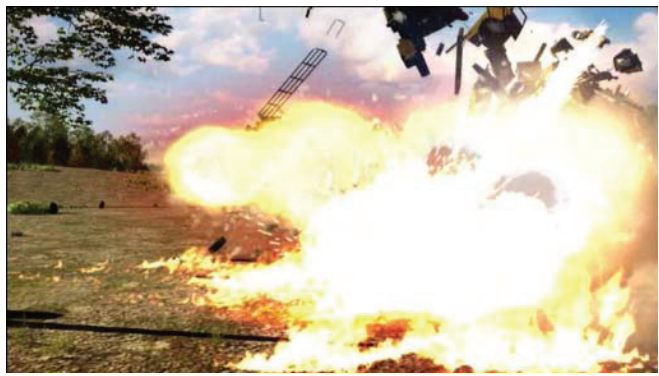

Figure 5. The effect of blast damage

\section{Concluding remarks}

Under the new situation, how to break the traditional teaching mode, focus on actual combat, adapt the training of ammunition talents to the requirement of construction and development at information age and carry out experimental teaching reformation of ammunition technology as well as combat utilization is an important project faced by military ammunition applications and security work. This paper bases on the virtual reality technology, and utilizes standardized software and hardware to construct a simulation platform which combines the technology and tactics to present the rocket action process. Results show that the simulation model of the platform is authentic and efficient, and the visualization effect is good, besides, the results possess high degree of simulaiton. The system has laid a solid foundation for the establishment and development of teaching resources and application abilities for virtual simulation teaching platform aiming at follow-up model rockets.

\section{References}

1. Guida M., Lanzotti A.Improving design validation of playground equipment in virtual reality[J].Internatio -al Journal on Interactive Design and Manufacturing, 2013,7(3):191-201. 
2. Lim C.K., Chen I.M., Yeo S.H.A virtual reality syste $-\mathrm{m}$ for arm and hand rehabilitation [J].Frontiers of $\mathrm{M}$ echanical Engineering,2011,6(1):347-354.

3. J Rigelsford.Virtual Reality Technology[J].Presence, 2015,12(6):663-664.

4. ZM Aghajan, L Acharya, JJ Moore.Impaired spatial selectivity and intact phase precession in two dimensional virtual reality[J].Nature Neuroscience, 2015,18(1):121

5. W Wang, Z Lv, X Li, W Xu.Virtual Reality Based GIS Analysis Platform[J].International Conference on Neural Information, 2015:638-645

6. C Chou, H Hsu, Y Yao.Construction of a virtual reality learning environment for teaching structural analysis[J].Computer Applications in Engineering Education,2015,5(4):223-230

7. RI Garcíabetances, MTA Waldmeyer, G Fico.A Succinct Overview of Virtual Reality Technology Use in Alzheimer's Disease[J].Frontiers in Aging Neuroscience, 2015,7:80

8. E Ronchi, D Nilsson.A Virtual Reality Experiment o n Flashing Lights at Emergency Exit Portals for Roa d Tunnel Evacuation[J].Fire Technology,2016, 52(3): 623-647

9. A Moglia, V Ferrari, L Morelli.A Systematic Review of Virtual Reality Simulators for Robotassisted Surgery[J].European Urology,2016,69(6):1065-1080

10. Castelvecchi, Davide.Low-cost headsets boost virtual reality's lab appeal[J].Nature,2016,533:153

11. A Alaraj, CJ Luciano, DP Bailey.Virtual Reality Cer e-bral Aneurysm Clipping Simulation With Real Tim e Haptic Feedback[J].Neurosurgery,2015,11,2(1):52

12. Webel.Evaluating virtual reality and augmented reality training for industrial maintenance and assembly tasks[J].Interactive Learning Environments, 2015,23(6):778-798 